\title{
Challenges, Strategies and Adaptations on Interactive Dashboards
}

DOI:

$10.1145 / 3340631.3398678$

$10.1145 / 3340631.3398678$

Document Version

Accepted author manuscript

Link to publication record in Manchester Research Explorer

\section{Citation for published version (APA):}

Alhamadi, M. (2020). Challenges, Strategies and Adaptations on Interactive Dashboards. In UMAP 2020

Proceedings of the 28th ACM Conference on User Modeling, Adaptation and Personalization (pp. 368-371).

(UMAP 2020 - Proceedings of the 28th ACM Conference on User Modeling, Adaptation and Personalization).

Association for Computing Machinery. https://doi.org/10.1145/3340631.3398678, https://doi.org/10.1145/3340631.3398678

Published in:

UMAP 2020 - Proceedings of the 28th ACM Conference on User Modeling, Adaptation and Personalization

\section{Citing this paper}

Please note that where the full-text provided on Manchester Research Explorer is the Author Accepted Manuscript or Proof version this may differ from the final Published version. If citing, it is advised that you check and use the publisher's definitive version.

\section{General rights}

Copyright and moral rights for the publications made accessible in the Research Explorer are retained by the authors and/or other copyright owners and it is a condition of accessing publications that users recognise and abide by the legal requirements associated with these rights.

\section{Takedown policy}

If you believe that this document breaches copyright please refer to the University of Manchester's Takedown Procedures [http://man.ac.uk/04Y6Bo] or contact uml.scholarlycommunications@manchester.ac.uk providing relevant details, so we can investigate your claim.

\section{OPEN ACCESS}




\title{
Challenges, Strategies and Adaptations on Interactive Dashboards
}

\author{
Mohammed Alhamadi \\ mohammed.alhamadi@manchester.ac.uk \\ The University of Manchester
}

\begin{abstract}
Interactive dashboards enable viewing and interacting with complex underlying data using visualisations such as charts, tables, maps, or even text typically on a single display. By bringing the most important information in a single place, dashboards enable performance monitoring and support decision making. Although nowadays dashboards are widely adopted in many domains, they involve challenges that prevent users from utilising them as they were intended. For example, having a dashboard with too much data can negatively affect decision making and lead to misleading interpretation. Through this research, we identify and investigate the challenges associated with dashboards, what users do in response to those challenges, and what adaptations can be applied to mitigate these challenges. Consequently, we aim to examine and evaluate a set of adaptation techniques that can improve the experience of users interacting with dashboards.
\end{abstract}

\section{CCS CONCEPTS}

- Human-centered computing $\rightarrow$ Information visualization; User models; Laboratory experiments; Activity centered design.

\section{KEYWORDS}

Dashboards, interaction challenges, information presentation, user modelling, adaptive interaction, user strategies, adaptations

ACM Reference Format:

Mohammed Alhamadi. 2020. Challenges, Strategies and Adaptations on Interactive Dashboards. In Proceedings of the 28th ACM Conference on User Modeling, Adaptation and Personalization (UMAP '20), fuly 14-17, 2020, Genoa, Italy. ACM, New York, NY, USA, 4 pages. https://doi.org/10.1145/ 3340631.3398678

\section{RESEARCH PROBLEM}

With the sheer amount of openly available data nowadays, a proliferation of visualisation dashboards has increased in nearly every industry [28]. Dashboards are used in several domains such as learning analytics, medicine, manufacturing, energy, commerce, news, software development, environment, and social media analysis among other domains. In its simplest form, a dashboard supports

Permission to make digital or hard copies of all or part of this work for personal or classroom use is granted without fee provided that copies are not made or distributed for profit or commercial advantage and that copies bear this notice and the full citation on the first page. Copyrights for components of this work owned by others than the author(s) must be honored. Abstracting with credit is permitted. To copy otherwise, or republish, to post on servers or to redistribute to lists, requires prior specific permission and/or a fee. Request permissions from permissions@acm.org.

UMAP '20, fuly 14-17, 2020, Genoa, Italy

(C) 2020 Copyright held by the owner/author(s). Publication rights licensed to ACM. ACM ISBN 978-1-4503-6861-2/20/07 . \$15.00

https://doi.org/10.1145/3340631.3398678 presenting and making sense of complex data to enable performance monitoring and support decision making. There are several definitions of dashboards in the literature. One of the most agreed upon is that of Few [13] where dashboards were defined as "a visual display of the most important information needed to achieve one or more objectives, consolidated and arranged on a single screen so the information can be monitored at a glance." There is no general agreement, however, on a definition for a dashboard, which is attributed to the expansion of data and visualisation technologies increasing dashboards adoption to new domains, which led to evolution of the dashboard concept [28, 29]. Recently and in response to coronavirus (COVID-19) pandemic, many interactive dashboards have been developed by world health agencies $[8,25,37]$. These dashboards are mainly intended for media and public use. However, their interaction and information presentation techniques vary widely.

Researchers in the field are in general agreement that dashboards, although widely adopted, are still under-researched and under-explored [24, 28, 39]. The rising diversity and ubiquity of dashboards make it difficult to formulate universal dashboard design guidelines that can be widely adopted [28]. The lack of proper design guidelines has resulted in the development of many dashboards that involve many challenges. Most dashboards are poorly designed displays and most of them fail due to designers focusing on making them aesthetically appealing more than functionally effective $[1,14]$. As a result, dashboards involve challenges related to the dashboard's main players: users, designers, and data. For each one of these, certain prerequisites must be met to have an effective dashboard. For example, users are challenged by the absence or the inefficiency of some functionalities needed to be more productive on the dashboard while designers are expected to possess good knowledge about several aspects of dashboards and visualisations. As for data, being the dashboard's main focus, it must be, for example, complete (without missing values).

Dashboards are useful for summarising data and alleviating information overload by utilising robust visualisation principles. They are used to improve performance, enhance business operations, improve strategic decision making, ease the integration of institutional data with decision making practices, improve routine monitoring, track processes effectiveness, minimise data complexity, communicate organisations' values to diverse stakeholders, support data-driven decision making, and enable real-time monitoring of dynamically updating data $[10,23,26,28]$. Our work focuses on the challenges users face when interacting with dashboards. This will inform our understanding and enable us to introduce adaptations to mitigate the challenges, which is still an open research problem [28]. Specifically, what challenges users face, what strategies they employ, and what adaptation techniques can be introduced to 
overcome these challenges. Our ultimate aim is to help inform the design of more effective dashboards.

\section{RELATED WORK}

We have looked at papers discussing challenges, strategies and adaptations in dashboards. There is almost an absence of works in the literature that link these three dashboard aspects. Adaptation, however, is not the only method of changing a dashboard to meet users' changing requirements. Vázquez-Ingelmo et al. [35] conducted a systematic mapping of the literature to understand the status of tailored information dashboards. They differentiate between customised, personalised, adaptive, and hybrid dashboards. They explain that customisation is done by the user to fit the interface, content, functionalities, etc. to their needs, personalisation is done by the system using user information and without user involvement at the dashboard creation stage, adaptive dashboards use information about the user to adapt the dashboard at run-time and may change as user's requirements change, and finally hybrid dashboards are personalised solutions with customisation support. Although adaptive dashboards can be argued to be more user-friendly since they require the least user involvement, they appear the least in literature (2 times; 9\%). Moreover, the authors identified only 23 works (in total) in the last 12 years (until 2018). The last three years (2016-2018) saw 57\% of the works), which is evidence that research is needed in the area. Below, we investigate the most relevant adaptive and personalised dashboard works to our research.

Dabbebi et al. [18] introduce a model to dynamically generate learning analytic dashboards based on the use context and adapt them if the context changes. They consider ten use contexts based on surveys with potential users. However, users, no matter how experienced in the domain they are, do not have enough visual and analytic literacy to determine what information is needed to achieve a task or the appropriate type of visualisation to represent the data even with standardised guidelines [10, 17, 36]. Belo et al. [3] proposed a method to adapt dashboards using a multi-agent system. The re-structuring agents frequently analyse a data log of the user's interactions collected in a session then define data to be shown at the next session. It learns from user's customisation events such as changing a visualisation element or its data through association discovery rules. The method, however, was not evaluated and the identification of users' preferences from the logs was not described in detail.

Toreini et al. [34] investigated the impact of attention and working memory limitation on dashboard effectiveness through an eyetracking study. They found that dashboards must adapt to user's visuospatial working memory capacity and should also adapt chart positions based on their importance. However, this study only investigates a single challenge (working memory capacity) and the dashboard used in the study is not interactive. Kintz et al. [21] use a model-driven approach to generate user-specific dashboards based on user roles. This approach, however, only provides a solution to the user adaptability challenge (adapting the dashboard to user's goal).

It can be seen that works on adaptive dashboards lack the connection between users' interaction strategies, adaptation techniques, and dashboard challenges. It is important to know this relationship as the strategies employed by dashboard users are indicators of problems and can inform the adaptation techniques to use. The contribution of our work lies in the identification of dashboard challenges, understanding dashboards user behaviour around them, finding relationships between challenges, strategies, and adaptations, and finally introducing adaptation techniques based on the found patterns.

\section{RESEARCH QUESTIONS AND PROPOSED APPROACH}

Dashboards have a variety of challenges as we will explain in more detail in the next section. Our research focuses on users' challenges with the interaction and information presentation aspects of dashboards. We also investigate user interaction strategies and adaptation techniques. There is not enough research on dashboards in general and dashboard adaptation, as opposed to other forms of tailoring, in particular. We propose using adaptation techniques to solve interaction and information presentation challenges on interactive dashboards informed by these challenges. Thus, in the scope of our research, we would like to seek answers to the following research questions:

(1) RQ1: What are the interaction and information presentation challenges users encounter when they interact with dashboards?

(2) RQ2: What are the adaptations that can be applied on dashboards and data tables? Which of these adaptations can be applied to address the problems found in RQ1.

(3) RQ3: How can we intervene to adapt the user interface? Do the adaptations improve the interaction with dashboards?

To tackle these questions we propose the following methodology:

(1) We have conducted a systematic literature review (results are in the next section) to identify dashboard challenges (any factor that limits the utilisation of dashboards). We have also identified users' strategies and adaptation techniques introduced by designers in response to these challenges.

(2) We will conduct a series of experiments to isolate what strategies users apply when faced with known interaction and information presentation challenges on dashboards. According to our experimental design, participants will interact with dashboards to complete a set of information finding tasks on dashboards that contain challenges and ones that do not. We will measure the efficiency (time taken to accomplish the required tasks), effectiveness (accuracy of task accomplishment), and participants' cognitive load using an eye-tracking device. We will collect self-reported qualitative data to ascertain their preferences and perceived challenges and to learn more about their mental models. We will look for patterns of use in user interaction with dashboards, which will be parts of user models. The collected data will be in the form of lowlevel event data where we will use WevQuery [2] to analyse it. The identified user models will inform our understanding of users' interaction strategies and help us adapt dashboards better, which was proved achievable [31].

(3) To understand how adaptation techniques are designed and built into dashboards, we will conduct interviews with dashboard development experts in several sectors (private and 
public sectors, non-governmental organisations, and academic and research institutions). Moreover, we will present the findings from the first study to the experts to discuss if the identified strategies are considered in the development process and whether they can help introduce robust adaptation techniques to interactive dashboards. We aim to conduct semi-structured interviews with questions designed before the interview but with the possibility to deviate if needed.

(4) Informed by the findings from previous stages, we aim to design adaptation techniques and implement them into interactive dashboards following a user-centered methodology. Finally, we will evaluate these techniques in a controlled user experiment.

\section{CURRENT PROGRESS AND FUTURE WORK}

To answer RQ1, we have conducted a systematic literature review to identify dashboard challenges without a focus on a dashboard type, domain, or users. Also, we have identified users' strategies and adaptation techniques introduced by designers in response to these challenges. The papers investigated were chosen pseudo-systematically. All of the reviewed works were retrieved from searches in Google Scholar and digital libraries such as ACM Digital Library, IEEE Xplore, Springer, and dblp. The search keywords included ("Dashboards" OR ("Dashboard AND challenges") OR ("Dashboard AND use AND challenges") OR ("Dashboard AND design AND challenges”) OR "Dashboard adaptation”). The retrieved papers' titles, abstracts, and keywords were examined for relevance to dashboard challenges, strategies, and adaptations. The inclusion criteria were: (1) does the paper describe a dashboard design, tool, review, etc.? (2) Is the paper about information dashboards (not other kinds) (3) Is it about visualisations used in dashboards? and (4) Is it written in English? Then for each of the selected works, the references were examined since they have high chances of relevance.

After removing the duplicate papers, we extracted dashboard challenges, strategies, and adaptations from the resulting corpus of papers $(\mathrm{N}=32)$. Most of the analysed papers were about dashboard system design $[4,6,9,11,15,16,19,20,22,24,32,33]$, then dashboard literature review $[5,7,12,29,39]$, dashboard user modelling [38], understanding dashboards [22, 24, 26, 28], or lab studies on high information density artefacts $[17,27,30]$. Although the lab studies did not look at the visualisation artefacts (tables, charts, etc.) in the context of dashboards, it was relevant to include them to understand challenges in their construction [17] and use [27, 30] because they are substantially used in dashboards.

We performed thematic analysis on the findings, by categorising the emerging themes under three a-priori themes:

(1) Challenges: any problem that hinders the use of dashboards.

(2) User strategies: any activity pattern employed by the user as a response to a challenge.

(3) Adaptation techniques: any technical intervention introduced by developers to mitigate a challenge.

We used another four a-priori codes to categorise the identified challenges [28]:

(1) User flexibility challenges: the absence or inefficiency of any dashboard feature that users need to use dashboards effectively.
(2) Visual, analytic, and data literacy challenges: any requirement of users or designers to use or develop effective dashboards, respectively.

(3) Data design challenges: any issue with the underlying data that hinders the utilisation of dashboards.

(4) Social impact challenges: socio-technical challenges related to how dashboards are perceived or their effects on people personally or socially.

As for user strategies and adaptation techniques, they were linked to the challenges as they are typically associated with them so there was no need to look into their emergence. The identified dashboard challenges, that fall under challenges theme, are as follows:

User Flexibility Challenges. These are: dashboard design and customisation, data detail adjustment, user adaptability, display adaptability, situation adaptability, data ink maximisation, comparison support, system integration, annotation and storytelling, and search functionality. For example, data detail adjustment is a feature that provides a summary of the data in a way that covers the most important points needed to aid the decision with the ability to explore more details on demand.

Visual, Analytic, and Data Literacy Challenges. The challenges here include: visual literacy, analytic literacy, data literacy, ease of use, designers knowledge, and dashboard evaluation. For example, dashboard evaluation challenge involves standardising methods of evaluating the effectiveness and efficiency of dashboards and putting them into use.

Data Design Challenges. This kind of challenges includes: data oversimplification, data quality, data order and grouping, data and metadata, too much data, data sources, inaccurate data representation, and key performance indicators and metrics. For example, the effects of low data quality such as incorrect or missing data can lead to misleading interpretation of data.

Social Impact Challenges. These challenges include: data-driven thinking, technology resistance, automation, added value, foresight deficiency, dashboard demotivation, quality costs, and data sharing, security, and privacy. For example, the challenge of added value occurs when knowledgeable dashboard users feel that no additional insight is gained from visualising the data and do not see added value of the dashboard they may not be inclined to use it.

We have identified a total of 32 challenges in the literature. We have also identified 59 adaptation techniques and 9 user strategies. An example of an adaptation for display adaptability challenge when display size changes we can use iconisation or remove less important charts. An example of a user strategy occurs when data drill down feature is absent. In that case, users seek more granular data elsewhere and keep switching back and forth between applications. Some of the identified challenges have as many as 9 adaptations but no strategies (data sources challenge), some have several adaptations and strategies (analytic literacy has 5 of each), most challenges have 3 adaptations/strategies on average, but 11 challenges have none at all. We can see that mapping between challenges, strategies, and adaptations is non-existent. Also, user strategies are especially lacking. Adaptations are introduced by designers to change some aspect of the dashboard in response to a 
dashboard challenge. On the other hand, users employ strategies to overcome challenges with dashboards. So dashboard challenges, adaptation techniques, and user strategies are interconnected as Figure 1 shows. The rationale is that if we are able to establish a relationship between strategies to problems and we can detect the strategies in real-time, we can adapt the user interface in realtime or in the next iteration. Then we can say that the adaption techniques have a positive effect on the challenges.

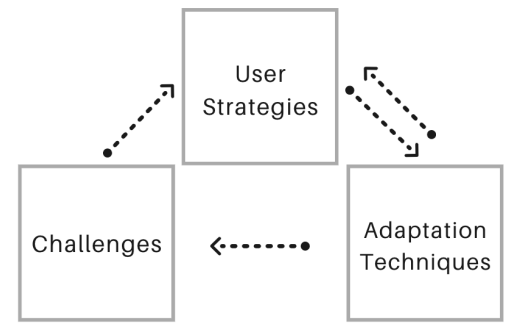

Figure 1: The relationship between dashboard challenges, user strategies, and adaptation techniques in our research.

The next step in our research is to conduct the dashboard user interaction strategies study to identify the links between the challenges and strategies. This paper has explained the progress done in the first six months of this $\mathrm{PhD}$ project. The tentative plan for the remainder of the project is as follows:

- Year 1: conduct dashboard user interaction strategies study.

- Year 1-2: interview dashboard designers.

- Year 2: design and implement the adaptation techniques.

- Year 2-3: evaluate the adaptation techniques.

- 2nd part of Year 3: write up the PhD thesis.

\section{REFERENCES}

[1] Ünal Aksu, Adela del Río-Ortega, Manuel Resinas, and Hajo A Reijers. 2019. An Approach for the Automated Generation of Engaging Dashboards. In OTM Confederated International Conferences" On the Move to Meaningful Internet Systems". Springer, 363-384.

[2] Aitor Apaolaza and Markel Vigo. 2017. WevQuery: Testing hypotheses about web interaction patterns. PACM on Human-Computer Interaction 1, EICS (2017), $1-17$.

[3] Orlando Belo, Paulo Rodrigues, Rui Barros, and Helena Correia. 2014. Restructuring dynamically analytical dashboards based on usage profiles. In International Symposium on Methodologies for Intelligent Systems. Springer, 445-455.

[4] Palash Bera. 2016. How colors in business dashboards affect users' decision making. Commun. ACM 59, 4 (2016), 50-57.

[5] Robert Bodily, Judy Kay, Vincent Aleven, Ioana Jivet, Dan Davis, Franceska Xhakaj, and Katrien Verbert. 2018. Open learner models and learning analytics dashboards: a systematic review. In LAK18. 41-50.

[6] Richard Brath and Michael Peters. 2004. Dashboard design: Why design is important. DM Direct 85 (2004), 1011285-1.

[7] Sandra C Buttigieg, Adriana Pace, and Cheryl Rathert. 2017. Hospital performance dashboards: a literature review. $7 \mathrm{HOM}$ (2017).

[8] CDC. 2020. COVIDView. Retrieved April 10, 2020 from cdc.gov/coronavirus/2019 ncov/covid-data/covidview/

[9] Sven Charleer, Kathrin Gerling, Francisco Gutiérrez, Hans Cauwenbergh, Bram Luycx, and Katrien Verbert. 2018. Real-time dashboards to support esports spectating. In ACM CHI PLAY 2018. 59-71.

[10] Aprisa Chrysantina and Johan Ivar Sæbø. 2019. Assessing User-Designed Dashboards: A Case for Developing Data Visualization Competency. In ICT4D 2019. Springer, 448-459.

[11] Arianna Dagliati, Lucia Sacchi, Valentina Tibollo, Giulia Cogni, Marsida Teliti, Antonio Martinez-Millana, Vicente Traver, Daniele Segagni, Jorge Posada, Manue Ottaviano, et al. 2018. A dashboard-based system for supporting diabetes care Journal of the American Medical Informatics Association 25, 5 (2018), 538-547.
[12] Dawn Dowding, Rebecca Randell, Peter Gardner, Geraldine Fitzpatrick, Patricia Dykes, Jesus Favela, Susan Hamer, Zac Whitewood-Moores, Nicholas Hardiker, Elizabeth Borycki, et al. 2015. Dashboards for improving patient care: review of the literature. Int. 7. Med. Inform. 84, 2 (2015), 87-100.

[13] Stephen Few. 2006. Information dashboard design: The effective visual communication of data. O'Reilly Media, Inc.

[14] Stephen Few. 2013. Information Dashboard Design: Displaying data for at-a-glance monitoring. Vol. 81. Analytics Press Burlingame, CA.

[15] Maria-Elena Froese and Melanie Tory. 2016. Lessons learned from designing visualization dashboards. IEEE CG\&A 2016 36, 2 (2016), 83-89.

[16] Sukumar Ganapati. 2011. Key features for designing a dashboard. Government Finance Review (2011), 0883-7856.

[17] Lars Grammel, Melanie Tory, and Margaret-Anne Storey. 2010. How information visualization novices construct visualizations. IEEE TVCG 2010 16, 6 (2010), 943-952.

[18] Dabbebi Ines, Iksal Sebastien, Gilliot Jean-Marie, May Madeth, and Garlatti Serge. 2017. Towards Adaptive Dashboards for Learning Analytic. (2017).

[19] Vladimir Ivanov, Vladislav Pischulin, Alan Rogers, Giancarlo Succi, Jooyong Yi, and Vasilii Zorin. 2018. Design and validation of precooked developer dashboards. In Proceedings of the 2018 26th ACM ESEC/FSE. 821-826.

[20] Andrea Janes, Alberto Sillitti, and Giancarlo Succi. 2013. Effective dashboard design. Cutter IT fournal 26, 1 (2013), 17-24.

[21] Maximilien Kintz, Monika Kochanowski, and Falko Koetter. 2017. Creating Userspecific Business Process Monitoring Dashboards with a Model-driven Approach.. In MODELSWARD. 353-361.

[22] Rob Kitchin, Sophia Maalsen, and Gavin McArdle. 2016. The praxis and politics of building urban dashboards. Geoforum 77 (2016), 93-101.

[23] Devender Maheshwari and Marijn Janssen. 2014. Dashboards for supporting organizational development: principles for the design and development of public sector performance dashboards. In ACM ICEGOV '14. 178-185.

[24] Ricardo Matheus, Marijn Janssen, and Devender Maheshwari. 2018. Data science empowering the public: Data-driven dashboards for transparent and accountable decision-making in smart cities. Government Information Quarterly (2018).

[25] NHS. 2020. UK COVID-19. Retrieved April 10, 2020 from gov.uk/government/ publications/covid-19-track-coronavirus-cases

[26] Koen Pauwels, Tim Ambler, Bruce H Clark, Pat LaPointe, David Reibstein, Bernd Skiera, Berend Wierenga, and Thorsten Wiesel. 2009. Dashboards as a service: why, what, how, and what research is needed? fournal of service research 12,2 (2009), 175-189.

[27] Charles Perin, Romain Vuillemot, and Jean-Daniel Fekete. 2014. À Table! improving temporal navigation in soccer ranking tables. In CHI '2014. 887-896.

[28] A. Sarikaya, M. Correll, L. Bartram, M. Tory, and D. Fisher. 2019. What Do We Talk About When We Talk About Dashboards? IEEE TVCG 2019 25, 1 (Jan 2019), 682-692.

[29] Beat A Schwendimann, María Jesús Rodríguez-Triana, Andrii Vozniuk, Luis P Prieto, Mina Shirvani Boroujeni, Adrian Holzer, Denis Gillet, and Pierre Dillenbourg. 2016. Understanding learning at a glance: An overview of learning dashboard studies. In LAK16. 532-533.

[30] Arjun Srinivasan, Matthew Brehmer, Bongshin Lee, and Steven M Drucker. 2018. What's the Difference? Evaluating Variations of Multi-Series Bar Charts for Visual Comparison Tasks. In CHI '2018. 1-12.

[31] Ben Steichen, Michael MA Wu, Dereck Toker, Cristina Conati, and Giuseppe Carenini. 2014. Te, Te, Hi, Hi: Eye gaze sequence analysis for informing useradaptive information visualizations. In UMAP 2014. Springer, 183-194.

[32] Dragos Strugar. 2019. Complex Systems: On Design and Architecture of Adaptable Dashboards. In TOOLS 2019. Springer, 176-186.

[33] Henri Tokola, Christoph Gröger, Eeva Järvenpää, and Esko Niemi. 2016. Designing manufacturing dashboards on the basis of a Key Performance Indicator survey. Procedia CIRP 57 (2016), 619-624.

[34] Peyman Toreini and Moritz Langner. 2019. Designing user-adaptive information dashboards: Considering limited attention and working memory. In Proceedings of European Conference on Information Systems (ECIS 2019). 08-14.

[35] Andrea Vázquez-Ingelmo, Francisco José García-Peñalvo, and Roberto Therón. 2019. Tailored information dashboards: A systematic mapping of the literature. In Interacción 2019. 1-8.

[36] Simon Wakeling, Paul Clough, James Wyper, and Amy Balmain. 2015. Graph literacy and business intelligence: Investigating user understanding of dashboard data visualizations. Business Intelligence fournal 20, 4 (2015), 8-19.

[37] WHO. 2020. WHO Health Emergency Dashboard. Retrieved April 10, 2020 from who.sprinklr.com/

[38] Ainhoa Yera, Javier Muguerza, Olatz Arbelaitz, Iñigo Perona, Richard N Keers, Darren M Ashcroft, Richard Williams, Niels Peek, Caroline Jay, and Markel Vigo. 2019. Modelling the interactive behaviour of users with a medication safety dashboard in a primary care setting. Int. f. Med. Inform. 129 (2019), 395-403.

[39] Ogan M Yigitbasioglu and Oana Velcu. 2012. A review of dashboards in performance management: Implications for design and research. International fournal of Accounting Information Systems 13, 1 (2012), 41-59. 